\title{
Beziehungen zwischen Planktonentwicklung und Bakterien im Vierwaldstättersee und Rotsee
}

\author{
Von ERNSt SchegG, EAWAG, Zürich \\ Vorläufige Mitteilung
}

Manuskript eingegangen am 31. Mai 1968

Auf eine enge Verkoppelung von Phytoplankton und Gewässerbakterien weist Overbeck $[1,2,3,4]$ in verschiedenen Arbeiten hin. In einem geschichteten See treten sowohl im Vertikalprofil als auch in der zeitlichen Entwicklung der Bakterien grosse Schwankungen auf. OvERBEck [4] betont, dass dieses geschichtete Vorkommen der Bakterien nur so zu erklären ist, dass das vom Phytoplankton durch Exkretion und Autolyse freigesetzte organische Material am Ort der Entstehung rasch wieder von Bakterien inkorporiert wird. Zwangsläufig müsste dann die Bakteriendichte sowohl in räumlicher als auch in zeitlicher Hinsicht mit der vorhandenen Phytoplanktonbiomasse einerseits und mit deren Stoffwechselleistung (Primärproduktion) andererseits übereinstimmen.

Im folgenden werden die Ergebnisse eines streng parallel geführten Vergleichs zwischen Phytoplanktonpopulation, Primärproduktion und Bakteriengesamtzahlen mitgeteilt. Die Untersuchungen wurden vom Februar bis Juni 1967 am leicht eutrophierten Vierwaldstättersee (Stelle Kreuztrichter, Tiefe $110 \mathrm{~m}$ ) und am polytrophen Rotsee (Tiefe $15 \mathrm{~m}$ ) bei Luzern durchgeführt ${ }^{1}$ ).

\section{Methodik}

Neben der Bestimmung der Phytoplanktonbiomasse nach UTERMöHL [5] wurde die Primärproduktion mittels der ${ }^{14} \mathrm{C}$-Methode nach Steeman-Nielsen [6] bestimmt. Die Totalzahl der Bakterien wurde durch Direktzählung nach RAzumow [7] auf Membranfiltern ermittelt. Dabei wurde die Erfahrung gemacht, dass sich die besten mikroskopischen Bilder erzielen lassen, wenn man die mit Erythrosin gefärbten Präparate im Phasenkontrastverfahren auszählt.

1) Für die Felduntersuchungen stand mir das der EAWAG angeschlossene Hydrobiologische Laboratorium der ETH in Kastanienbaum zur Verfügung. Herrn Prof. Dr. O. Jaag und Herrn Dr. H. Ambühl, Leiter dieses Laboratoriums, sei dafür herzlich gedankt. 


\section{Ergebnisse}

Bei der Auswertung wurde vor allem der dynamische Gesichtspunkt in den Vordergrund gerückt, im Bewusstsein der Schwierigkeiten, diese Vorgänge mit relativ wenigen Messungen wirklich als Kontinuum zu erfassen.

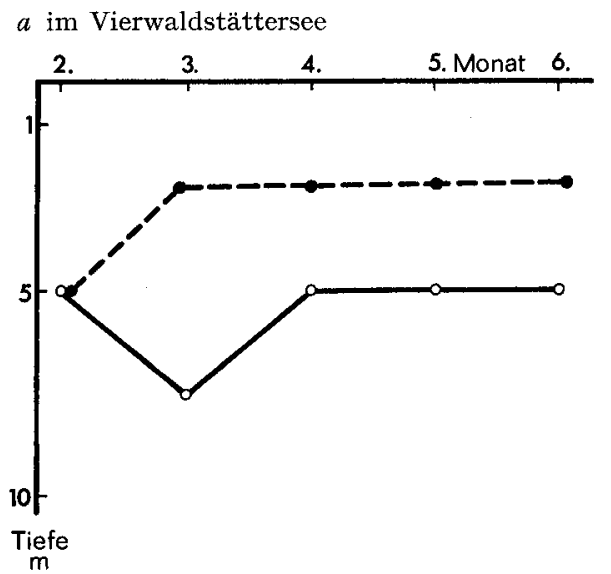

$b$ im Rotsee

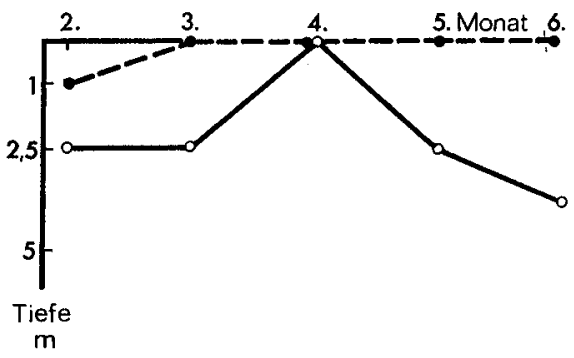

Abb. 1. Relative Lage der Maxima der Primärproduktion (- $-\rightarrow-)$ und der Gesamtkeimzahl $(\longrightarrow)$ in der trophogenen Schicht.

In den Abbildungen $1 a$ und $b$ wurden die Primärproduktionsmaxima und die Maxima der Bakterien bezüglich ihrer räumlichen und zeitlichen Verteilung aufgetragen. Es zeigt sich, dass in beiden Fällen die Maximalzahlen der Bakterien immer mittelbar unterhalb oder in der Schicht der höchsten Primärproduktion liegen. Diese Koppelung ist um so enger, je höher der Trophiegrad des zu untersuchenden Gewässers ist. Daraus resultiert die Vermutung, dass in einem geschichteten Gewässer der kleine Kreislauf in der trophogenen Schicht den leicht resorbierbaren Teil der frei werdenden organischen Substanz umsetzt. Nur damit lässt sich die gefundene räumliche Verteilung der hauptsächlich aeroben heterotrophen Bakterien erklären.

Wenn ein grosser Teil der für Bakterien substratbildenden Stoffe in der trophogenen Schicht verbleibt, müsste der Anfall der Nährstoffe für die Bakterien des Hypolimnions mit einer zeitlichen Verschiebung auftreten. Dies lässt sich aus den hypolimnischen Bakterienzahlen einerseits und dem $\mathrm{CO}_{2}$-Einbau durch die Bakterienpopulation andererseits feststellen.

In Abb. 2 wird die gesamte in der Wassersäule vorhandene Biomasse mit der Zahl der hypolimnischen Bakterien verglichen.

Die Bakterienzahlen im Hypolimnion des Rotsees steigen erst langsam, dann beschleunigt an, während die Phytoplanktonentwicklung schon früher ihr Maximum erreicht. Beachtenswert ist auch, dass der Gehalt an Sauerstoff in $14 \mathrm{~m}$ Tiefe erst gegen Mai völlig aufgezehrt wird.

Nach SoRokiN [8] bauen auch die heterotrophen Bakterien einen Teil ihrer organischen Substanz durch direkte Carboxylierung aus $\mathrm{CO}_{2}$ auf. Die Stoffwechselleistung 
einer Mischpopulation kann also auch mittels des Einbaus von ${ }^{14} \mathrm{CO}_{2}$ bestimmt werden. Betrachtet man die ${ }^{14} \mathrm{C}$-Hellwerte und die ${ }^{14} \mathrm{C}$-Dunkelfixierung des Hypolimnions $(14 \mathrm{~m})$ aus dem Rotsee (Tab. 1), so lassen sich diese Zusammenhänge ebenfalls feststellen.

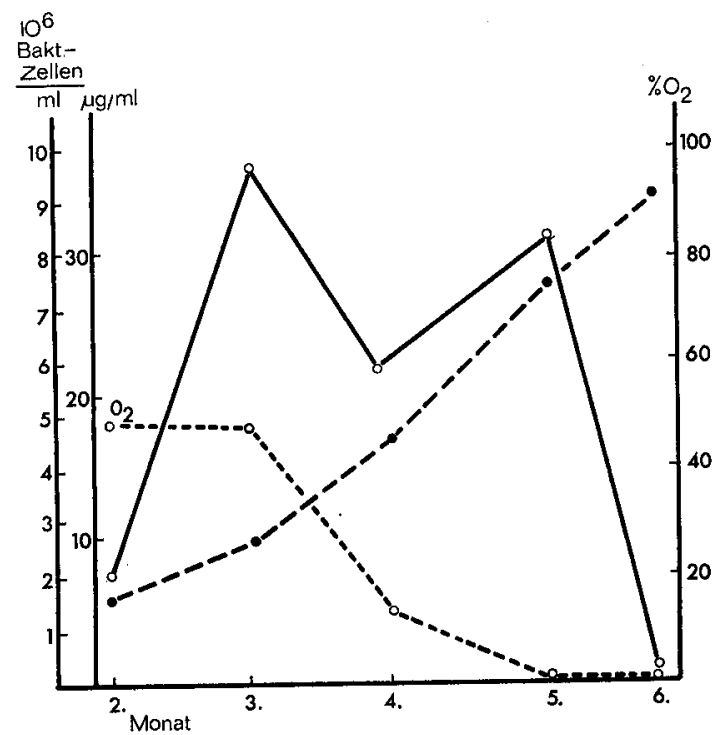

Abb. 2. Vergleich der Phytoplanktonbiomasse (___ - ) in der trophogenen Schicht mit den Bakterienzahlen (_- _ $)$ und der Sauerstoffsättigung (_.......... im Hypolimnion im Rotsee.

Tab. 1 Vergleich der ${ }^{14} \mathrm{C}$-Hellwerte in $0 \mathrm{~m}$ Tiefe und der ${ }^{14} \mathrm{C}$-Fixierung durch Bakterien in $14 \mathrm{~m}$ Tiefe (Hypolimnion) aus dem Rotsee.

\begin{tabular}{rcc}
\hline Monat & \multicolumn{2}{c}{${ }^{14} \mathrm{C}$-Fixierung in $\mathrm{mgC} / \mathrm{m}^{3} \cdot \mathrm{h}$} \\
& $0 \mathrm{~m}$ "Hell" & $14 \mathrm{~m}$ "Dunkel" \\
\hline 24.2. & 52,3 & 2,2 \\
22.3. & 171,8 & 1,0 \\
18.4. & 584,1 & 0,8 \\
18.5. & 287,6 & 4,4 \\
8.6. & 10,3 & 3,3 \\
\hline
\end{tabular}

Aus Tab. 1 erkennt man, dass zur Zeit der grössten Primärproduktion in $0 \mathrm{~m}$ Tiefe die Fixierung der Bakterien im Hypolimnion ein Minimum aufweist.

Aus diesen Befunden lässt sich folgende Dynamik herauslesen: Der Abbau der anfallenden organischen Substanz wird namentlich in der ersten Phase der Entwicklung (Februar bis April) zu einem grösseren Teil durch die aeroben heterotrophen Bakterien der trophogenen Schicht durchgeführt. Erst gegen Ende dieser Periode (Mai, Juni) fallen im Hypolimnion neben den schwerer resorbierbaren, fortlaufend sedimentierenden Substanzen grössere Mengen an Substrat an.

Der Nährstoffkreislauf in der trophogenen Schicht gerät zu dieser Zeit aus verschiedenen Gründen aus dem «Gleichgewicht». Es ist anzunehmen, dass beim son, 
merlichen Absterben des Phytoplanktons nicht nur der Nährstoffschwund infolge Überproduktion an Planktonorganismen, sondern auch die Akkumulierung der Nährstoffe in den grossen Bakterienmengen eine entscheidende Rolle spielt.

Die wichtige Phytoplankton-Bakterien-Relation soll in den Abb. 3 und 4 gezeigt werden. Die Kurven sind aus den gewogenen Mitteln der Einzelmessungen gewonnen und umfassen die trophogene Schicht. Die Beurteilung der beiden genannten Mittelwertsdarstellungen führt zu folgenden prinzipiellen Aussagen:

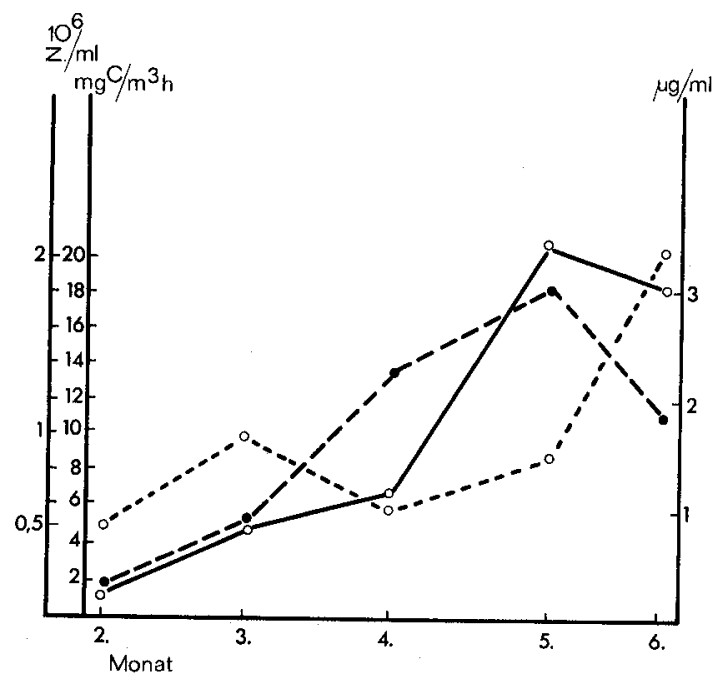

Abb. 3. Vierwaldstättersee. Mittlerer Gehalt an Balsterien $\left(10^{6}\right.$ Zellen/ml $(----\rightarrow)$. Mittlerer Gehalt an Phytoplanktonbiomasse $(\mu \mathrm{g} / \mathrm{ml})(\ldots-\ldots)$. Mittlere Primärproduktion $\left(\mathrm{mg} \cdot \mathrm{C} / \mathrm{m}^{3} \cdot \mathrm{h}\right)$ ).

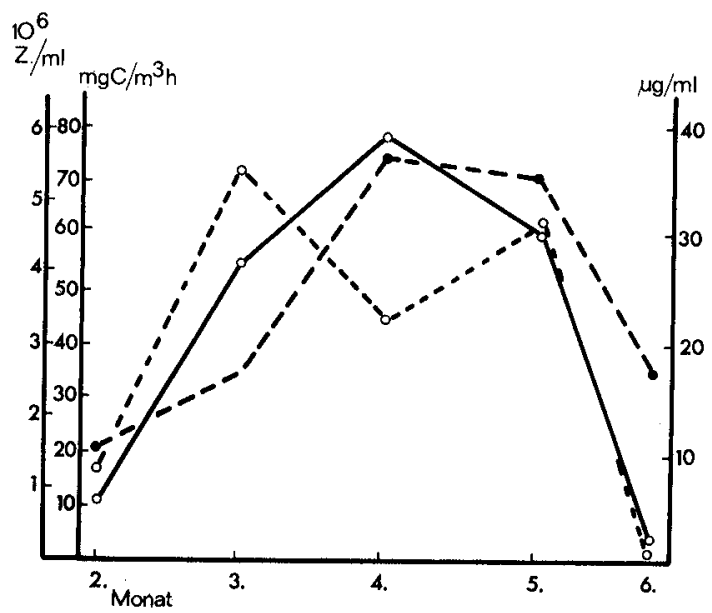

Abb. 4. Rotsee, Legende siehe Abb. 3. 
Zwischen der Entwicklung der Bakterienzahlen und den Werten der Primärproduktion besteht eine enge Beziehung, während zwischen vorhandener Phytoplanktonbiomasse und den Bakterienzahlen nur indirekte Zusammenhänge bestehen. Dies gilt sowohl für die Verhältnisse des Vierwaldstättersees als auch die des Rotsees.

Dass zwischen Primärproduktion und Bakterienzahlen eine feste Korrelation besteht, zeigt sich auch, wenn einzelne Tiefenstufen gesondert aufgetragen werden. Das eine angeführte Beispiel (Abb. 5) stammt aus dem Rotsee aus $0 \mathrm{~m}$ Tiefe.

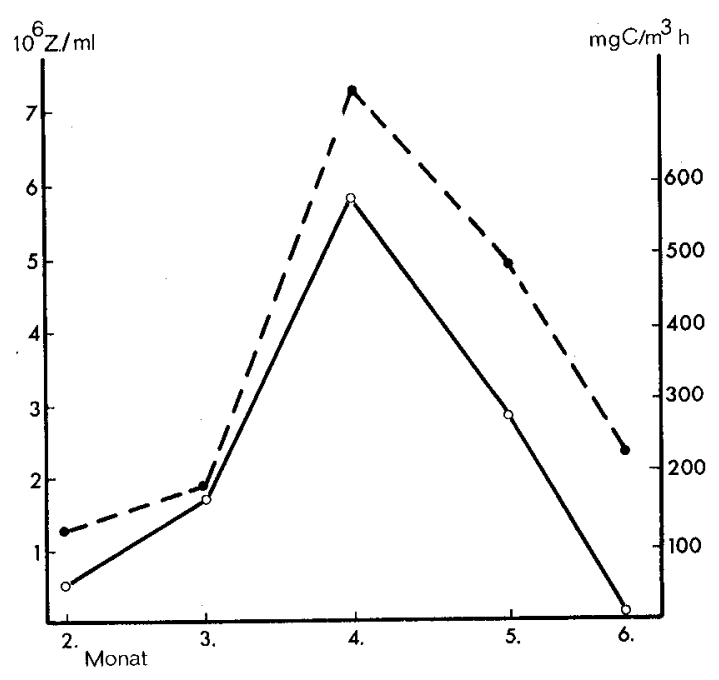

Abb. 5. Entwicklung der Bakterienzahlen (-- - - ) und der Primärproduktion (aus $0 \mathrm{~m}$ Tiefe des Rotsees.

Ein spezieller Fall, der aber diese Zusammenhänge recht schön demonstriert, wird in Abb. 6 geschildert. Es handelt sich um einen Vergleich der Messungen vom April und Mai in $0 \mathrm{~m}$ Tiefe im Rotsee. Die Planktonbiomasse verzeichnet einen Anstieg, während sich die Primärproduktion verringert. Es können weder Temperatur noch Witterungsverhältnisse dafür verantwortlich gemacht werden. Offenbar ist hier die Zusammensetzung des Planktons produktionsbestimmend. Tatsächlich bestand das Phytoplankton im April fast ausschliesslich aus Zellen von Chlorella sp. $(2,5 \mathrm{Mio} / \mathrm{ml})$, während einen Monat später die Planktonmasse zu $75 \%$ aus Oscillatoria oblique acuminata bestand.

In Übereinstimmung mit RovHe [9] wurde gefunden, dass kleinere Zellen mit einer grösseren aktiven Oberfläche $(4300: 60)$ eine höhere Produktionsleistung zu erbringen vermögen. Die entscheidende Frage ist nun: Wie verhalten sich die Bakterienzahlen in diesen verschiedenen Verhältnissen? Trotz kleinerer vorhandener Biomasse des Phytoplanktons korrelieren die Bakterienzahlen mit der Primärproduktion. 

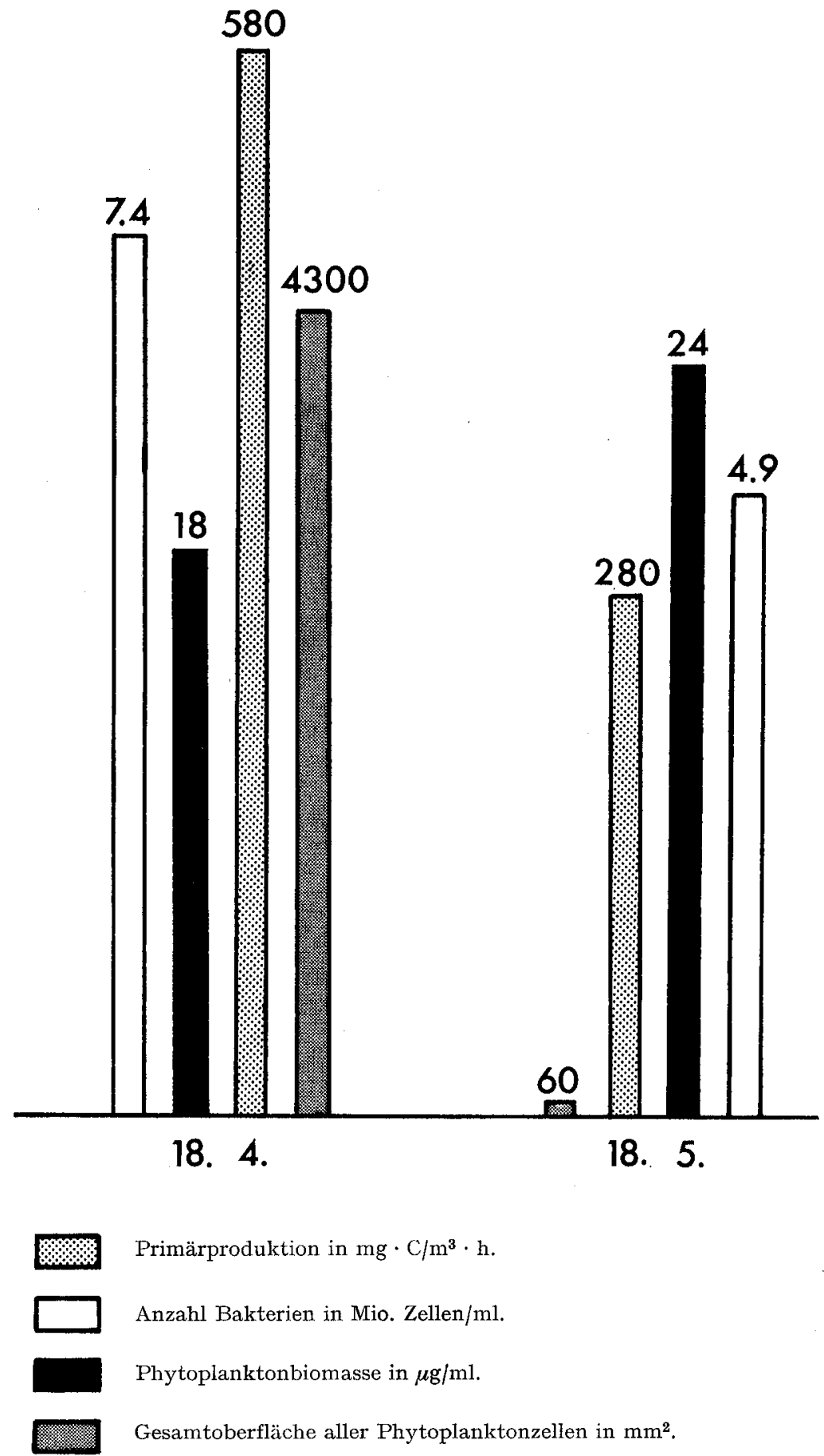

Abb. 6. Vergleich der beiden Messungen vom April und Mai 1967 aus dem Rotsee aus $0 \mathrm{~m}$ Tiefe. 


\section{Schlussfolgerungen}

Mit der vorliegenden Arbeit wurde ein Nachweis erbracht, dass die Entwicklung der Bakterienpopulation, insbesondere der heterotrophen Keime, direkt mit derjenigen des Phytoplanktons gekoppelt ist. Dieser Zusammenhang ist stofflicher Natur [4], d. h. die Bakterien leben von Autolyse und Exkretionsprodukten der Algen. Zu erwarten wäre, dass die Bakterienzahlen von der vorhandenen und damit früher oder später der Destruktion anheimfallenden Biomasse abhingen. Diese Beziehung besteht aber nur in zweiter Linie und dürfte ungefähr derjenigen zwischen Primärproduktion und "standing crop» entsprechen. Das Ergebnis deutet darauf hin, dass die laufende Exkretion einer Planktonpopulation, die ja mit der Stoffwechselleistung korreliert, einen bedeutenden substratbildenden Einfluss hat. Es handelt sich offenbar bei der Abhängigkeit der Bakterien von der Primärproduktion um ein wesentliches ökologisches Prinzip, dessen weitere Erforschung viele neue Aspekte des Stoffkreislaufs in einem Gewässer erschliessen wird.

\section{Zusammenfassung}

1. Es werden die Resultate einer Untersuchung über Beziehungen zwischen Phytoplankton- und Bakterienpopulation mitgeteilt. Die Untersuchungen wurden von Februar bis Juni 1967 am Vierwaldstättersee und am Rotsee bei Luzern durchgeführt.

2. Zwischen Primärproduktion und Bakterienzahlen in der trophogenen Schicht wurde eine feste Korrelation gefunden (Abb. 3-5).

3. Die gefundene Korrelation weist darauf hin, dass nicht nur die aus Autolyse anfallende Substanz, sondern auch die laufende Exkretion einen bedeutenden substratbildenden Einfluss hat.

\section{SUMMARY}

1. This report shows the results of an investigation about the relations between phytoplankton and bacterial populations in the lake of Lucerne and the Rotsee near Lucerne, from February to June 1967.

2. A close correlation between primary production and the number of bacteria was found in the trophogenic layer (Fig. 3-5).

3. This correlation points to the fact that not only the organic substances due to autolysis, but also the current excretion is of great influence as to the forming of new substrate.

\section{LITERATUR}

[1] Overbeck J., Babenzien H. D., Bahterien und Phytoplankton eines Kleingewässers im Jahreszyklus, Z. allg. Mikrobiol. 4, 59 (1964).

[2] Overbeck J., Primärproduktion und Gewässerbahterien, Naturwissenschaften 51, 145 (1965).

[3] Overbeck J., Neue Ergebnisse der Gewässerbakteriologie, Umschau 66, 474 (1966).

[4] Overbeck J., Zur Bahteriologie des Süsswassersees - Ergebnisse und Probleme, GWF 108, 1258 (1967).

[5] UteRMÖHL. H., Zuv Vervollkommnung der quantitativen Phytoplanktonmethodik, Mitt. int. Ver. theor. angew. Limnol. 9, 1 (1958). 
[6] Stemman-Nielsen E., The Use of Radio-active Carbon $\left({ }^{14} C\right)$ for Measuring of Organic Production in the Sea, J. Cons. 18, 117 (1952).

[7] Aus Kusnezow J., Die Rolle der Mikroorganismen im Stoffkreislauf der Seen, 50 (DVW, Berlin 1959).

[8] Sorokin J. U., On the Trophic Role of Chemosynthesis and Bacterial Biosynthesis in Water Bodies, Mem. Ist. ital. Idrobiol. Suppl. 18, 187 (1965).

[9] Rodhe W., The Primary Production in Lakes: Some Results and Restrictions of the ${ }^{14} \mathrm{C}$ Method, Cons, int. Explor. de la Mer, Rapp. et Proc.-Verb. 144, 122 (1958).

Adresse des Autors:

Ernst Schegg, dipl. sc. nat. ETH, EAWAG, Physikstr. 5, 8044 Zürich

Separatdruck aus der Schweiz. Zeitschrift für Hydrologie 30, Fasc. 2 (1968) Herausgegeben mit Unterstützung der Stiftung der Wirtschaft zur Förderung des Gewässerschutzes in der Schweiz 